\title{
ADMINISTRAÇÃO NA
}

\section{CONTEMPORANEIDADE:} RELATOS DE PESOUISA

\section{Volume 1}

Organizadores:

Milena Nunes Alves de Sousa

Larissa de Araújo Batista Suárez

Vera Lúcia Soares de Oliveira Claudino

Denilson Costa de Carvalho 


\section{ADMINISTRAÇÃO NA}

\section{CONTEMPORANEIDADE:} RELATOS DE PESOUISA

\section{Volume 1}

Organizadores:

Milena Nunes Alves de Sousa

Larissa de Araújo Batista Suárez

Vera Lúcia Soares de Oliveira Claudino

Denilson Costa de Carvalho 
Editora Omnis Scientia

ADMINISTRAÇÃO NA CONTEMPORANEIDADE: RELATOS DE PESQUISA

Volume 1

$1^{a}$ Edição

TRIUNFO - PE 


\section{Editor-Chefe}

Me. Daniel Luís Viana Cruz

Organizador (a)

Milena Nunes Alves de Sousa

Larissa de Araújo Batista Suárez

Vera Lúcia Soares de Oliveira Claudino

Denilson Costa de Carvalho

\section{Conselho Editorial}

Dra. Pauliana Valéria Machado Galvão

Dr. Wendel José Teles Pontes

Dr. Walter Santos Evangelista Júnior

Dr. Cássio Brancaleone

Dr. Plínio Pereira Gomes Júnior

Editores de Área - Ciências Sociais Aplicadas

Dra. Helga Midori Iwamoto

Dra. Milena Nunes Alves de Sousa

Dr. Thiago Barbosa Soares

\section{Assistentes Editoriais}

Thialla Larangeira Amorim

Andrea Telino Gomes

Imagem de Capa

Freepik

\section{Edição de Arte}

Leandro José Dionísio

\section{Revisão}

Os autores

\section{(2) $\oplus \Theta \Theta$}

Este trabalho está licenciado com uma Licença Creative Commons - Atribuição-NãoComercial-SemDerivações 4.0 Internacional.

O conteúdo abordado nos artigos, seus dados em sua forma, correção e confiabilidade são de responsabilidade exclusiva dos autores. 
Dados Internacionais de Catalogação na Publicação (CIP) (eDOC BRASIL, Belo Horizonte/MG)

A238 Administração na contemporaneidade [livro eletrônico] : relatos de pesquisa / Organizadores Milena Nunes Alves de Sousa... [et al.]. - Triunfo, PE: Omnis Scientia, 2021.

233 p. : il.

Formato: PDF

Requisitos de sistema: Adobe Acrobat Reader

Modo de acesso: World Wide Web

Inclui bibliografia

ISBN 978-65-88958-31-5

DOI 10.47094/978-65-88958-31-5

1. Administração de empresas. 2. Planejamento estratégico. 3. Empreendedorismo. I. Sousa, Milena Nunes Alves de. II. Suárez, Larissa de Araújo Batista. III. Claudino, Vera Lúcia Soares de Oliveira. IV. Carvalho, Denilson Costa de.

CDD 658.4

Elaborado por Maurício Amormino Júnior - CRB6/2422

Editora Omnis Scientia

Triunfo - Pernambuco - Brasil

Telefone: +55 (87) 99656-3565

editoraomnisscientia.com.br

contato@editoraomnisscientia.com.br 


\section{DEDICATÓRIA}

Dedicamos a todos aqueles, que como nós, amam a administração! 


\section{AGRADECIMENTOS}

Agradecemos aos nossos alunos e a Faculdade São Francisco da Paraíba por todo apoio. 


\section{SINOPSE}

Esta obra intitulada "Administração na Contemporaneidade: Relatos de Pesquisa", retrata o esforço de gestores, professores e alunos em realizarem estudos atuais na área de gestão. Portanto, traz em seu cerne uma coletânea de 19 artigos que abarcam as mais distintas temáticas.

Entre as abordagens, têm-se enfoques nas áreas de 1) Marketing, com capítulo sobre marketing pessoal; 2) Finanças, contemplando a educação financeira; 3) Empreendedorismo, abarcando a mulher no mercado de trabalho; 4) Recursos humanos, enfocando a comunicação interpessoal nas organizações, liderança, recrutamento e seleção de pessoas; 5) Planejamento estratégico, como o capítulo sobre a implantação de ferramentas do planejamento estratégico em uma empresa do setor varejista; 6) Administração geral, com pesquisas sobre gestão em organizações do terceiro setor, criatividade e inovação, desafios organizacionais no mundo digital, bem com gestão escolar democrática e outros; e por fim, 7) Gestão da Produção, com estudos sobre planejamento da gestão da produção, sobre a curva $\mathrm{ABC}$ e classificação XYZ, por exemplo.

Esperamos que este compêndio seja uma valorosa fonte de consulta e de transmissão de saberes!

Os organizadores 


\section{ORGANIZADORES}

Milena Nunes Alves de Sousa - Turismóloga, Administradora e Enfermeira. Especialista em Gestão e Análise Ambiental, Saúde da Família, Saúde Coletiva, Enfermagem do Trabalho, Docência em Enfermagem, Docência e Pesquisa para a Área de Saúde, Formação em Coaching, Administração Estratégica, Administração e Auditoria em Serviços de Saúde, Metodologias Ativas e Enfermagem em Oncologia. MBA em Liderança Sustentável e Coaching Executivo. Mestrado em Ciências da Saúde. Doutorado e Pós-Doutorado em Promoção de Saúde. Pós-Doutorado em Sistemas Agroindustriais pela Universidade Federal de Campina Grande (UFCG), Campus Pombal, Paraíba. Pró-Reitora de Pesquisa, Extensão e Pós-Graduação no Centro Universitário de Patos (UNIFIP). Docente no Curso de Medicina do UNIFIP, Patos, Paraíba. Docente na Faculdade São Francisco da Paraíba (FASP), Cajazeiras-PB, Brasil. E-mail: minualsa@gmail.com.br

Orcid: 0000-0001-8327-9147

Larissa de Araújo Batista Suárez - Administradora e Psicóloga. Doutoranda e Mestre em Psicologia Clínica pela Universidade Católica do Pernambuco (UNICAP). Especialista em Psicologia Organizacional, Psicopedagogia Institucional, Gestão de Marketing, Gestão de Pessoas, Tutoria em EaD e Docência do Ensino Superior e Terapia Familiar. Consultora empresarial, Coordenadora do Curso de Administração e Docente na Faculdade São Francisco da Paraíba (FASP), Cajazeiras-PB e Docente na Faculdade São Francisco do Ceará (FASC), Iguatu, Ceará, Brasil. E-mail: labsuarez@gmail.com.

Orcid: 0000-0002-6658-5019

Vera Lúcia Soares de Oliveira Claudino - Bióloga. Mestrado em Educação. Diretora Presidente da Faculdade São Francisco da Paraíba (FASP), Cajazeiras, Paraíba. Diretora Presidente da Faculdade São Francisco do Ceará (FASC), Iguatu, Ceará, Brasil. E-mail: veraluciaclaudino@bol.com.br

Orcid: 0000-0001-5292-9725

Denilson Costa de Carvalho - Administrador. Mestre em Engenharia de Produção pela Universidade Federal da Paraíba. Professor no Curso de Graduação em Administração da Faculdade São Francisco da Paraíba (FASP), Cajazeiras, Paraíba, Brasil. E-mail: denilsoncarvalho.sdm@gmail.com.

Orcid: 0000-0003-3366-9889 


\section{AUTORES}

Afrânio Souto Duque de Abrantes - Mestre em Sistemas Agroindustriais pela Universidade Federal de Campina Grande. Professor da Faculdade de Filosofia, Ciências e Letras de Cajazeiras. Professor do Curso de Bacharelado em Administração da Faculdade São Francisco da Paraíba (FASP), Cajazeiras, Paraíba, Brasil. E-mail: afranioabrantes@fsf.edu.br.

Aleska Ketley Feitosa Ferreira - Graduada em Administração pela Faculdade São Francisco da Paraíba (FASP), Cajazeiras, Paraíba, Brasil. E-mail: aleskaferreira@gmail.com.

Ana Paula Pinheiro da Silva - Especialista em Docência do Ensino Superior - IFCE, Doutoranda em Ciência e Engenharia de Materiais (UFSCAR), São Carlos, São Paulo, Brasil. E-mail: anapaulapinheirosilva@estudante.ufscar.br.

André Luiz Dantas Bezerra - Enfermeiro e Cirurgião Dentista. Mestre em Sistemas Agroindustriais. É estudante na Residência Multiprofissional em Atenção Primária à Saúde pelo Centro Universitário de Patos (UNIFIP). Atualmente é professor do curso de Administração da Faculdade São Francisco da Paraíba (FASP) e do Instituto Superior de Educação de Cajazeiras (ISEC), Cajazeiras, Paraíba, Brasil. E-mail: dr.andreldb@gmail.com.

Brenna Maria Moura Germano - Graduada em Administração pela Faculdade São Francisco da Paraíba (FASP), Cajazeiras, Paraíba, Brasil. E-mail: brennamaria96@gmail.com.

Bruno Stéfano de Medeiros Plácido - Graduado em Administração pela Faculdade São Francisco da Paraíba (FASP), Cajazeiras, Paraíba, Brasil. E-mail:bruno_placido@yahoo.com.br.

Cleilson Morais da Silva - Graduado em Administração pela Faculdade São Francisco da Paraíba (FASP), Cajazeiras, Paraíba, Brasil. E-mail: cleilson2380@gmail.com.

Cynthia Monielly de Souza - Graduada em Administração pela Faculdade São Francisco da Paraíba (FASP), Cajazeiras, Paraíba, Brasil. E-mail: moniellycynthia@gmail.com.

Danilo de Sousa Cezario - Mestrado em Educação pela Universidade Internacional Três Fronteiras. Doutorando em Ciências da Religião pela Universidade Católica de Pernambuco (UNICAP). Atualmente é professor do curso de Administração da Faculdade São Francisco da Paraíba (FASP) e do Instituto Superior de Educação de Cajazeiras (ISEC), Cajazeiras, Paraíba, Brasil. E-mail: daniloscezario@hotmail.com.

Denilson Costa de Carvalho - Mestre em Engenharia de Produção pela Universidade Federal da Paraíba. Professor no Curso de Graduação em Administração da Faculdade São Francisco da Paraíba (FASP), Cajazeiras, Paraíba, Brasil. E-mail: denilsoncosta@fsf.edu.br.

Edivânia Maria Leite da Silva - Graduada em Administração pela Universidade Federal da Paraíba. Especialista em Administração pela Fundação Getúlio Vargas. Mestrado em Sistemas Agroindustriais pela Universidade Federal de Campina Grande. Coordenadora da Pós-Graduação da Faculdade de 
Filosofia, Ciências e Letras da Paraíba (FAFIC). Professora na FAFIC e na Faculdade São Francisco da Paraíba (FASP), Cajazeiras, Paraíba, Brasil. E- Consultora e Instrutora do Serviço de Apoio às Micro e Pequenas Empresas (SEBRAE Paraíba e Pernambuco) nas áreas de Planejamento Empresarial, Empreendedorismo e Recursos Humanos. E-mail: edivaniasilva@fsf.edu.br.

Francisca Alves da Silva - Graduada em Letras. Professora Mestra do curso de Administração Faculdade São Francisco da Paraíba (FASP), Cajazeiras, Paraíba, Brasil. E-mail: neidinhaalves@fsf. edu.br.

Jael Marla de Souza Albuquerque - Graduada em Administração pela Faculdade São Francisco da Paraíba (FASP), Cajazeiras, Paraíba, Brasil. E-mail: jaelalbuquerque@gmail.com

Jean Alencar de Oliveira - Possui graduação em Administração pelo Centro Universitário de João Pessoa (2001) e mestrado pela Universidade Potiguar (2010). Atualmente é professor do curso de Administração da Faculdade São Francisco da Paraíba (FASP), Cajazeiras, Paraíba, Brasil. E-mail: jeanalencar@fsf.edu.br.

Joandeson Lacerda de Oliveira - Graduado em Administração pela Faculdade São Francisco da Paraíba (FASP), Cajazeiras, Paraíba, Brasil. E-mail: joandeson_lacerdak3@hotmail.com.

José Ciedston Tomaz de Sousa Andrade - Graduado em Ciências, com habilitação em Matemática. Professor Mestre do Curso de Bacharelado em Administração da Faculdade São Francisco da Paraíba (FASP), Cajazeiras, Paraíba, Brasil. E-mail: ciedston@fsf.edu.br.

Josineuda Vieira de Freitas - Graduada em Administração pela Faculdade São Francisco da Paraíba (FASP), Cajazeiras, Paraíba, Brasil. E-mail:. josineudafreitas@hotmail.com.

Kelly Simonne Sarmento de Oliveira - Graduada em Administração pela Faculdade São Francisco da Paraíba (FASP), Cajazeiras, Paraíba, Brasil. E-mail: kelly_simonne18@hotmail.com.

Larissa de Araújo Batista Suárez - Administradora e Psicóloga. Mestre e Doutoranda em Psicologia Clínica pela Universidade Católica do Pernambuco (UNICAP). Coordenadora do Curso de Administração e Docente na Faculdade São Francisco da Paraíba (FASP), Cajazeiras-PB, Brasil. E-mail: labsuarez@gmail.com.

Leiana Isis Soares de Oliveira - Graduada em Administração pela Faculdade São Francisco da Paraíba (FASP), Cajazeiras, Paraíba, Brasil. E-mail: isysolliveira@gmail.com.

Luma Ronnielli Dias Menezes - Graduada em Administração pela Faculdade São Francisco da Paraíba (FASP), Cajazeiras, Paraíba, Brasil. E-mail: E-mail: lumamenezes@gmail.com.

Marcos Vinícius Lins Ferreira - Possui graduação em Administração pela Universidade Federal da Paraíba. Pós-graduação em Metodologia do Ensino e em Estatística Aplicada Mestrando do curso de Ciências da Educação pela Universidade Tecnológica Intercontinental (UTIC). Professor do Curso de Graduação em Administração da Faculdade São Francisco da Paraíba (FASP), Cajazeiras, Paraíba, 
Brasil. E-mail: marcoslins@fsf.edu.br.

Maria Aparecida Cavalcanti de Oliveira - Graduada em Administração pela Faculdade São Francisco da Paraíba (FASP), Cajazeiras, Paraíba, Brasil. E-mail: macavalcanti@gmail.com.

Milena Nunes Alves de Sousa - Turismóloga, Administradora e Enfermeira. Doutorado e Pós-Doutorado em Promoção de Saúde. Pós-Doutorado em Sistemas Agroindustriais. Docente no Curso de Medicina do Centro Universitário de Patos (UNIFIP) e Faculdade São Francisco da Paraíba (FASP). E-mail: minualsa@gmail.com.br.

Mirian de Sousa Lira Ferreira - Graduada em Administração pela Faculdade São Francisco da Paraíba (FASP), Cajazeiras, Paraíba, Brasil. E-mail: miriankm123@gmail.com.

Nethanya Kleysla Faustino Santos - Graduada em Administração pela Faculdade São Francisco da Paraíba (FASP), Cajazeiras, Paraíba, Brasil. E-mail: nethanyakfaustino@gmail.com.

Patrick Nobre da Silva - Bacharel em Ciências Contábeis pelo Centro Universitário do Rio Grade do Norte. Auditor Interno da Prefeitura Municipal de Cajazeiras. Professor do Curso de Bacharelado em Administração da Faculdade São Francisco da Paraíba (FASP), Cajazeiras, Paraíba, Brasil. E-mail: patricknobre@fsf.edu.br.

Pedro Ítalo Alexandre Coelho - Graduado em Administração pela Faculdade São Francisco do Ceará (FASC), Iguatú, Ceará, Brasil. E-mail: pedaoalexandre@gmail.com.

Rafael Andrade Lins de Almeida - Mestrado em Administração pela Universidade Potiguar. Professor do Curso de Bacharelado em Administração da Faculdade São Francisco da Paraíba (FASP), Cajazeiras, Paraíba, Brasil. E-mail: rafaellins@fsf.edu.br.

Robério Vieira de Menêses - Graduado em Administração pela Faculdade São Francisco da Paraíba (FASP), Cajazeiras, Paraíba, Brasil. E-mail: roberiocz2290@gmail.com.

Vera Lúcia Soares de Oliveira Claudino - Bióloga. Mestrado em Educação. Diretora Presidente da Faculdade São Francisco da Paraíba (FASP), Cajazeiras, Paraíba. Diretora Presidente da Faculdade São Francisco do Ceará (FASC), Iguatu, Ceará, Brasil. E-mail: veraluciaclaudino@bol.com.br. 


\section{SUMÁRIO}

CAPÍTULO 1

COMUNICAÇÃO INTERPESSOAL NAS ORGANIZAÇÕES: UM ESTUDO DE CASO REALIZADO EM INDÚSTRIA DA PARAÍBA

Kelly Simonne Sarmento de Oliveira

André Luiz Dantas Bezerra

Milena Nunes Alves de Sousa

DOI: 10.47094/978-65-88958-31-5/19-27

CAPÍTULO 2. 28

EMPODERAMENTO FEMININO: O PROCESSO DE EXPANSÃO DA MULHER NO MERCADO DE TRABALHO NA ÁREA ADMINISTRATIVA

Aleska Ketley Feitosa Ferreira

Marcos Vinícius Lins Ferreira

Milena Nunes Alves de Sousa

DOI: $10.47094 / 978-65-88958-31-5 / 28-36$

CAPÍTULO 3.

EMPREENDEDORISMO NA PERSPECTIVA DE ESTUDANTES DO CURSO DE

VESTUÁRIO DE CAJAZEIRAS-PB

Robério Vieira de Menêses

Edivânia Maria Leite da Silva

Milena Nunes Alves de Sousa

DOI: $10.47094 / 978-65-88958-31-5 / 37-44$ 
Maria Aparecida Cavalcanti de Oliveira

Larissa de Araújo Batista Suárez

Milena Nunes Alves de Sousa

DOI: $10.47094 / 978-65-88958-31-5 / 45-56$

CAPÍtUlO 5. 57

CRIATIVIDADE E INOVAÇÃO NAS EMPRESAS BRASILEIRAS

Pedro Ítalo Alexandre Coelho

Ana Paula Pinheiro da Silva

Larissa de Araújo Batista Suárez

DOI: 10.47094/978-65-88958-31-5/57-68

\section{CAPÍTULO 6}

GESTÃO DE ESTOQUES: UTILIZAÇÃO DAS FERRAMENTAS CURVA ABC E CLASSIFICAÇÃO XYZ EM SUPERMERCADO

Joandeson Lacerda de Oliveira

José Ciedston Tomaz de Sousa Andrade

Rafael Andrade Lins de Almeida

DOI: 10.47094/978-65-88958-31-5/69-83

CAPÍTULO 7 .84

IMPLANTAÇÃO DE FERRAMENTAS DO PLANEJAMENTO ESTRATÉGICO EM UMA EMPRESA DO SETOR VAREJISTA

Mirian de Sousa Lira Ferreira

Patrick Nobre da Silva

Rafael Andrade Lins de Almeida

DOI: 10.47094/978-65-88958-31-5/84-97 
CUSTO DE ARMAZENAGEM: ESTUDO DE CASO NA LERIO'S DISTRIBUIDORA DE BEBIDAS DE SANTA CRUZ-PB

Cleilson Morais da Silva

Afrânio Souto Duque de Abrantes

Rafael Andrade Lins de Almeida

DOI: 10.47094/978-65-88958-31-5/98-110

CAPÍTULO 9. 111

ESTUDO SOBRE A SATISFAÇÃO NO PÓS VENDA DOS CLIENTES DE LOJA VAREJISTA DA PARAÍBA

Josineuda Vieira de Freitas

Larissa de Araújo Batista Suárez

Jean Alencar de Oliveira

DOI: 10.47094/978-65-88958-31-5/111-116

CAPÍTULO 10.

PEQUENAS EMPRESAS E DESAFIOS NO MUNDO DIGITAL: ESTUDO EM

ORGANIZAÇÕES DE PEQUENO PORTE CEARENSES

Juscileudo Lucas Belo

Patrick Nobre da Silva

Denilson Costa de Carvalho

DOI: $10.47094 / 978-65-88958-31-5 / 117-125$

CAPÍTULO 11

PLANEJAMENTO DA GESTÃO DA PRODUÇÃO EM UMA CERÂMICA

Jael Marla de Souza Albuquerque 
Danilo de Sousa Cezario

Denilson Costa de Carvalho

DOI: 10.47094/978-65-88958-31-5/126-137

CAPÍTULO 12 138

FINANÇAS PESSOAIS: IMPORTÂNCIA DA EDUCAÇÃO FINANCEIRA PARA

DISCENTES DO CURSO DE ADMINISTRAÇÃO

Luma Ronnielly Dias Menezes

Afrânio Souto Duque de Abrantes

Denilson Costa de Carvalho

DOI: 10.47094/978-65-88958-31-5/138-145

CAPÍTULO 13 146

RECRUTAMENTO E SELEÇÃO DE PESSOAS COM DEFICIÊNCIA E A

RESPONSABILIDADE SOCIAL NAS EMPRESAS

Myllene Dantas da Silva

Denilson Costa de Carvalho

Larissa Araújo Batista Suárez

DOI: 10.47094/978-65-88958-31-5/146-158

CAPÍTULO 14. 159

EDUCAÇÃO FINANCEIRA COMO ESTRATÉGIA DE MINIMIZAÇÃO DE IMPACTOS CAUSADOS PELO COVID-19

Brenna Maria Moura Germano

Patrick Nobre da Silva

José Ciedston Tomaz de Sousa Andrade

DOI: 10.47094/978-65-88958-31-5/159-170 
LIDERANÇA COMO DIFERENCIAL ESTRATÉGICO NO BANCO DO BRASIL

Bruno Stéfano de Medeiros Plácido

Larissa de Araújo Batista Suárez

Milena Nunes Alves de Sousa

DOI: 10.47094/978-65-88958-31-5/171-180

CAPÍTULO 16. 181

RELEVÂNCIA DA CONSTRUÇÃO DO MARKETING PESSOAL: ESTUDO COM CONCLUINTES DO CURSO DE ADMINISTRAÇÃO

Leiana Isis Soares de Oliveira

Larissa de Araújo Batista Suárez

Milena Nunes Alves de Sousa

DOI: 10.47094/978-65-88958-31-5/181-192

CAPÍTULO 17.

PERFIL DAS MULHERES EMPREENDEDORAS DE BARRO-CE E OS IMPACTOS DA COVID-19 NOS SEUS NEGÓCIOS

Nethanya Kleysla Faustino Santos

Vera Lúcia Soares de Oliveira Claudino

Francisca Alves da Silva

DOI: 10.47094/978-65-88958-31-5/193-204

CAPÍTULO 18. .205

AVALIAÇÃO DO USO DO HOME OFFICE NO AMBIENTE VIRTUAL DE ENSINO E APRENDIZAGEM DOS DOCENTES DO CURSO DE BACHARELADO EM ADMINISTRAÇÃO

Cynthia Monielly de Souza 
Milena Nunes Alves de Sousa

Denilson Costa de Carvalho

DOI: 10.47094/978-65-88958-31-5/205-214

CAPÍTULO 19

DESAFIOS E BENEFÍCIOS DA GESTÃO ESCOLAR DEMOCRÁTICA: UM MODELO EM QUE AS VANTAGNES SOBRESSAEM AOS DESAFIOS

Danilo de Sousa Cezario

Milena Nunes Alves de Sousa

Vera Lúcia Soares de Oliveira Claudino

DOI: 10.47094/978-65-88958-31-5/215-226 


\title{
CAPÍTULO 10
}

\section{PEQUENAS EMPRESAS E DESAFIOS NO MUNDO DIGITAL: ESTUDO EM ORGANIZAÇÕES DE PEQUENO PORTE CEARENSES}

\author{
Juscileudo Lucas Belo \\ Patrick Nobre da Silva \\ Denilson Costa de Carvalho
}

\section{INTRODUÇÃO}

A tecnologia tem se mostrado bastante eficaz quando usada de forma estratégica à comunicação entre empresa e clientes. O número de empresas que utilizam a internet para procurar por produtos e serviços disparou sem precedentes nas últimas décadas, indo de encontro ao alarmante número de pessoas, os potenciais clientes, que a utilizam.

Quando se fala sobre a conectividade que o marketing digital proporciona, Kotler (2017, p. 34), diz que "ela diminui de forma significativa os custos de interação entre empresas, funcionários, parceiros de canal, clientes e outras partes envolvidas”. Portanto, as empresas buscam, através do marketing digital, promover seu diferencial competitivo e, devido às mudanças aceleradas no mercado, precisam estar em constante desenvolvimento e aprimoramento das suas ferramentas de comunicação para possibilitar a construção de um relacionamento direto e transparente com o cliente.

As empresas podem obter vasto relacionamento com os clientes através do uso de colaboradores que se utilizem das ferramentas para contatar com seus consumidores. Para Teixeira (2010), uma pequena empresa, em sua maioria, não tem condições de manter um profissional especializado em marketing digital para gerir a área e que geralmente é uma função desempenhada pelo próprio proprietário. Logo, este tem sido o maior desafio enfrentado pelas pequenas empresas e, piora, se comparado às grandes corporações.

Para usufruir do marketing digital com eficiência e eficácia, se faz necessário que as empresas retenham mais conhecimento do seu público-alvo e buscar trabalhar de maneira mais personalizada, pois beneficia a empresa com a coleta de dados obtida dos clientes e se crie campanhas publicitárias de acordo com seu nicho de mercado.

Torres (2010, p.8) aconselha que se "pense quem é o seu consumidor. Aquele que de fato precisa e dá valor ao seu produto. O seu cliente ideal". Com isto, a empresa irá buscando alcançar a fidelização dos clientes, bem como irá, como consequência, contribuir com os resultados à empresa.

Com as redes sociais, geralmente, os clientes compartilham suas experiências de compra e as 
empresas que investem em seu Marketing digital estão contribuindo, mais do que nunca, com o diálogo entre a empresa e os clientes, buscando gerar oportunidades e manter-se à frente dos concorrentes. Gansky (2011. p.3) comenta que "os novos negócios baseados em compartilhamento são reforçados e construídos sobre as mídias sociais".

O marketing de relacionamento proporciona que a empresa forneça informações ao usuário da sua rede sempre que desejar e obter um feedback rápido da empresa, uma vez que a internet proporciona que a empresa não necessite se deslocar geograficamente, bem como o seu cliente. Conforme o Serviço Brasileiro de Apoio às Micro e Pequenas Empresas (SEBRAE, 2017), a internet é indispensável para o posicionamento de marca e de conectividade com o mercado à oferta de produtos, serviços e relacionamento com o cliente, sem a preocupação com barreiras geográficas. Portanto, percebe-se que o relacionamento entre as empresas e seus clientes é algo crescente e mais eficiente. Esta acontece quando o cliente é ouvido e recebe as respostas da empresa de forma rápida, sem barreiras geográficas, bem como, uma empresa que deseja crescer, tem que construir relacionamentos sólidos com os consumidores, sendo algo proporcionado de forma eficaz quando se utiliza das ferramentas de marketing digital via internet.

A escolha pelo tema tem como propósito identificar as estratégias, as quais, as mídias sociais proporcionam às empresas, uma vez que, funcionam como um canal de comunicação entre empresa e consumidor atualmente e, por se tratar de um assunto de grande importância e em grande ascensão, percebe-se que tais meios passam por diversas mudanças constantemente e se faz necessário entender o seu desenvolvimento e desempenho às empresas que as utilizam.

O objetivo deste trabalho é avaliar a utilização das mídias sociais das pequenas empresas em Umari-CE.

\section{MATERIAIS E MÉTODOS}

Para proceder com os meios de execução da pesquisa objetivada, buscou-se de métodos científicos para sua validação. Quanto ao objetivo do estudo, os resultados foram extraídos através de análise descritiva. Segundo, Prodanov e Freitas (2013, p.52) a pesquisa descritiva acontece "quando o pesquisador apenas registra e descreve os fatos observados sem interferir neles. Visa a descrever as características de determinada população ou fenômeno ou o estabelecimento de relações entre variáveis". Logo, a pesquisa é descritiva porque ilustra por meio de gráficos que possibilitam o cruzamento das respostas pertinentes ao problema elencado na pesquisa.

Para alcançar o objetivo proposto neste trabalho, o procedimento técnico utilizado foi um estudo de caso, por permitir um melhor acompanhamento e a proximidade com um fenômeno estudado. Conforme Yin (2001 apud PRODANOV; FREITAS, 2013, p. 60), "quando envolve o estudo profundo e exaustivo de um ou poucos objetos de maneira que permita o seu amplo e detalhado conhecimento".

A presente pesquisa, do ponto de vista da abordagem, é do tipo quali-quantitativa. A mesma 
é considerada qualitativa, uma vez que, há uma interpretação lógica dos fenômenos e, segundo Flick (2013, p. 93): “[...] pode-se querer explorar um campo para descobrir algo novo", a fim de obter conhecimentos sobre um determinado assunto, bem como é quantitativa, uma vez que, serão apurados dados de forma numérica e estatística. "O objetivo aqui é controlar e padronizar o maior número possível de características do grupo; depois, as diferenças entre eles podem ser rastreadas à variável que você está estudando" (FLICK, 2013, p. 90).

Quanto à área de estudo, definiu-se o município de Umari - CE, localizado na Microrregião de Lavras da Mangabeira, Mesorregião do Centro-Sul Cearense. O universo da pesquisa é formado pelos pequenos e microempreendedores da cidade e os quais utilizavam das Redes Sociais e do Marketing Digital como canal de comunicação.

A amostra foi formada por 15 proprietários da cidade. Para obtenção das informações empregadas no estudo, foram aplicados questionários entre os meses de setembro a novembro de 2020. $\mathrm{O}$ meio utilizado à coleta de dados foi o questionário, em que se utilizou a ferramenta "Google Forms", para os pesquisados responderem as arguições. Neste questionário aplicado contém quatorze questões, estruturadas. As perguntas se dividem em questões de formação de perfil do entrevistado, seu empreendimento e às respostas, às quais, são objetivos da pesquisa.

\section{RESULTADOS E DISCUSSÃO}

A maioria era do sexo feminino $(60 \% / n=9)$ (Gráfico 1) e todos os comerciantes informaram a sua idade, contudo, a faixa etária mais prevalente foi 25-25 anos (60\%; n=9) (Gráfico 2), indicando que o público jovem está em alta na abertura e desenvolvimento tecnológico das empresas.

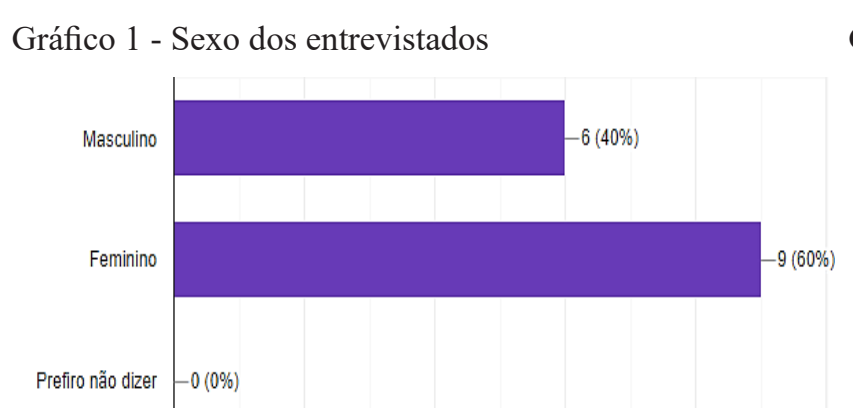

Fonte: Dados da pesquisa (2020).
Gráfico 2 - Idade dos entrevistados

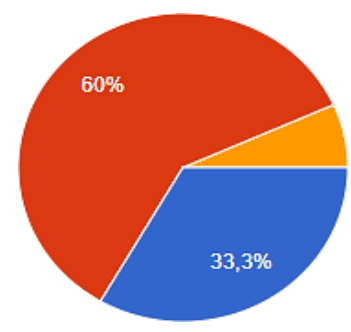

Fonte: Dados da pesquisa (2020).

As mídias sociais são cruciais, têm crescido constantemente e, dificilmente, retroagirá. Pelo contrário, as empresas, estão buscando novas e melhores maneiras de gerir seus negócios a partir das ferramentas digitais e têm logrado êxito, uma vez que, as mídias sociais têm proporcionado relacionamento entre empresa e cliente, pois faz parte da comunicação da empresa com o mercado.

Assim como afirma Twy (2012), quando diz que as pequenas empresas estão cada vez mais 
investindo na presença digital, utilizando-se de novas tecnologias das mais variadas formas com o intuito de obter novos cliente e divulgação da marca. A presente pesquisa demonstrou que é um fato, uma vez que, a maioria dos respondentes possui, não somente loja física, mas também virtual. Conforme o gráfico 3, a maioria respondeu possuir loja "física" e "virtual" (46,7\%; n=7), o que evidencia que a maioria dos empreendedores está mesclando entre o on-line e o presencial.

Quando a idade da loja física, 66,7\% (n=10) responderam "entre 1 e 5 anos", o que significa que a maioria das empresas não tem muito tempo de mercado e que ainda pode setar em introdução ou crescimento (Gráfico 4).

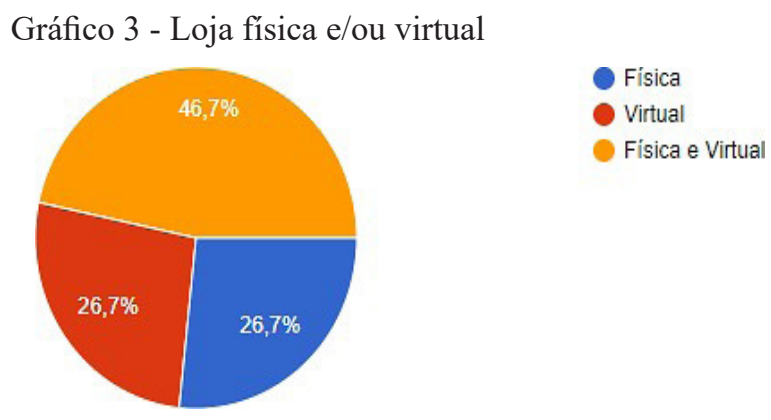

Fonte: Dados da pesquisa (2020).
Gráfico 4 - Idade da loja física

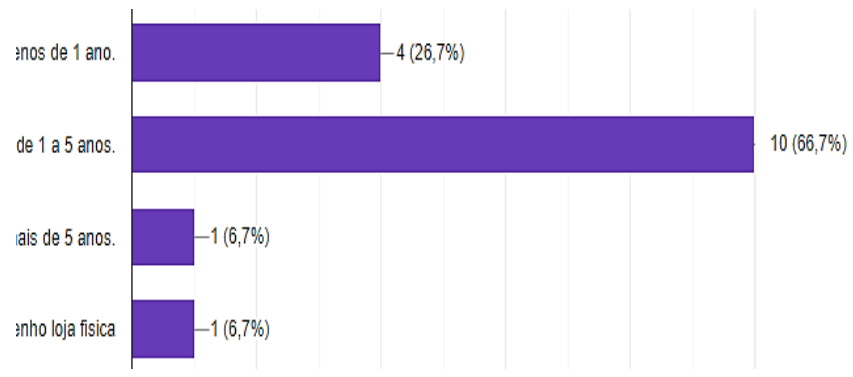

Fonte: Dados da pesquisa (2020).

Foi o perguntado aos comerciantes quais os meios utilizados à divulgação do negócio, se com parcerias e influenciadores; divulgação em loja virtual ou anúncios pagos, conforme o gráfico 5. A maiora disse fazer divulgação na loja virtual $(93,3 \%$; $=14)$.

No tocante as redes sociais que os comerciantes se utilizam à divulgação do negócio, perguntando-se quais das três redes sociais mais populares são utilizadas (Gráfico 6). Destaque para o "Instagram", ou seja, já que 100\% $(\mathrm{n}=15)$ a utilizam. Tal resultado demostra que todos utilizam ao menos uma rede social e a sua preferencia são essas plataformas, pois podem expor fotos, ideal para divulgação dos produtos.

Gráfico 5 - Meios de divulgação do negócio

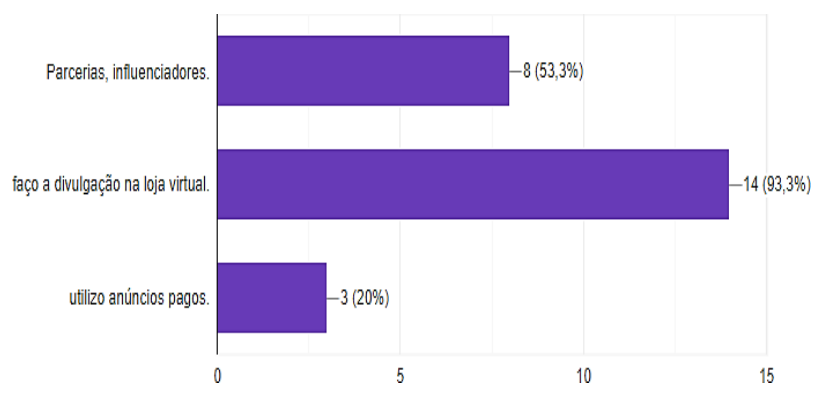

Fonte: Dados da pesquisa (2020).
Gráfico 6 - Redes sociais utilizadas no negócio

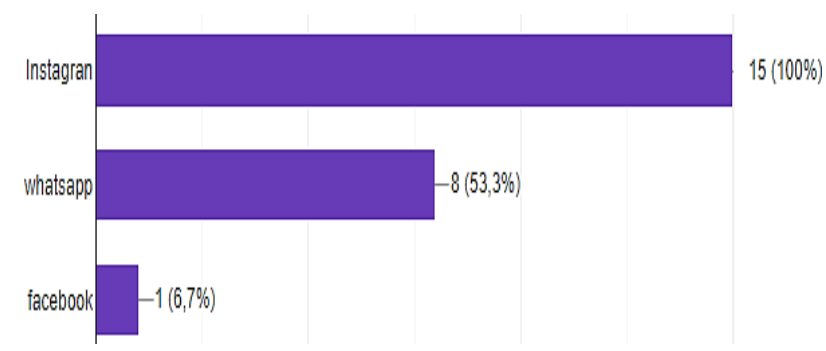

Fonte: Dados da pesquisa (2020).

Conforme Franca (2011), grandes organizações têm alocado cada vez mais investimento fi- 
nanceiro para o marketing digital à medida que as têm tornado mais consistentes e populares, porém às pequenas empresas essa não é a realidade, por conta do modesto ou quase nenhum investimento nas ferramentas virtuais.

Apesar disso, $100 \%(\mathrm{n}=15)$ dos respondentes disseram que utilizam as redes sociais para obter resultados nas vendas, o que demostra a sua relevância e importância nos tempos atuais (Gráfico 7). Em relação à utilização de anúncios pagos, a maioria não faz uso dessa estratégia $(73,3 \%$; n=11) (Gráfico 8) e aqueles que fazem anúncios pagos, investem somente $\mathrm{R} \$ 50,00(\mathrm{n}=4)$. O resultado demostra que os empreendedores digitais buscam ferramentas gratuitas de divulgação (FRANCA, 2011).

Gráfico 7 - Resultados proporcionado pelas redes sociais

Gráfico 8 - Utilização de anúncios pagos

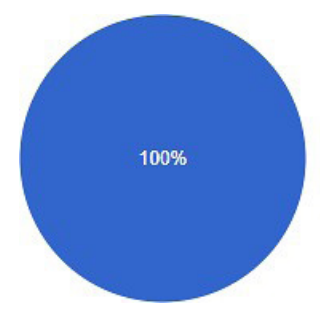

Fonte: Dados da pesquisa (2020).

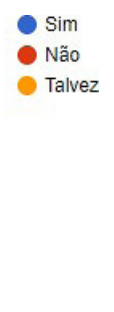

Não

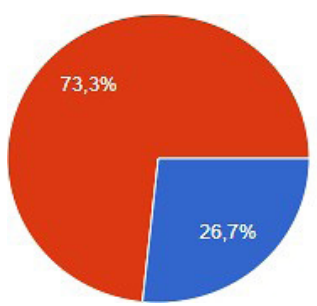

Fonte: Dados da pesquisa (2020).

Em relação ao engajamento que os clientes proporcionam à empresa, a maioria considerou como poderia ser melhor $(53,3 \% ; n=8)$ (Gráfico 9). O resultado mostra que os comerciantes esperam mais das redes sociais em relação ao engajamento dos clientes, o que demostra confiança.

Gráfico 9 - Engajamento dos clientes

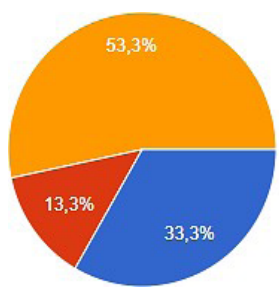

- Sim

- Talves.

Acho que poderia ser melhor

กล̃o.

Fonte: Dados da pesquisa (2020).

Foi perguntado aos comerciantes se os clientes ao comprarem o produto possuem o híbito de postar e marcar a loja nas redes sociais. Portanto, 10 (66,7\%) empreendedores responderam que "às vezes" (Gráfico 10). O resultado mostra que, apesar dos empreendedores esperarem mais dos clientes em relação ao engajamento, os mesmos ainda não retribuem a interação como deveriam com postagens e marcação dos produtos nas redes sociais. Quanto à inspiração dos empreendedores em outros do ramo, 12 (n=80\%) disseram que "sim" (Gráfico 11), mostrando que a maioria se espelha em outros empreendedores. 
Investir em marketing digital é uma boa estratégia para pequenas empresas, bem como buscar por inspirações em outros empreendedores bem-sucedidos, também é uma forma de compreender o processo digital para aplicar na organização (TWY, 2012).

Gráfico 10 - Postagem e marcação dos produtos nas redes Gráfico 11 - Inspiração em outros empreendedores sociais após a compra

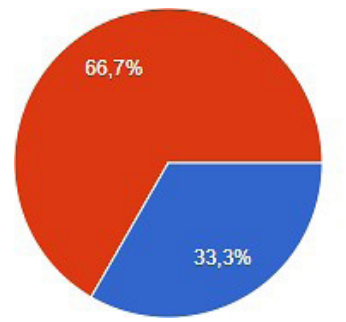

Fonte: Dados da pesquisa (2020).

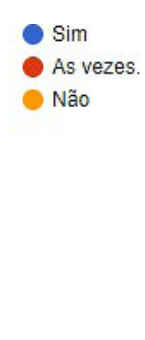

As vezes.

Não

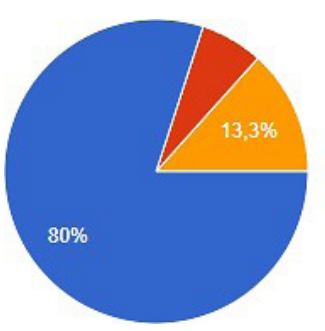

Fonte: Dados da pesquisa (2020).

Também foi indagado sobre o hábito dos empreendedores estudar sobre o marketing digital para melhoria do negócio. A maioria disse que $\operatorname{sim}(93,3 \%$; $n=14)$ (Gráfico 12), contudo, 33,3\% ( $\mathrm{n}=5$ ) que sempre que pode e $40 \%(n=6)$ afirmaram constantemente e $20 \%(n=3)$ "às vezes". Tal resultado indica falta de preparo por grande parte dos empreendedores em relação ao conhecimento sobre marketing digital. Twy (2012) afirma que para utilizar as ferramentas online de maneira responsável é necessário compreender o processo digital para aplicar na organização.

Por fim, questionou-se o meio de maior realização de vendas, se físico ou virtual (Gráfico 13). Logo, a "loja física" ainda é o principal canal $(53,3 \%$; $n=8)$.

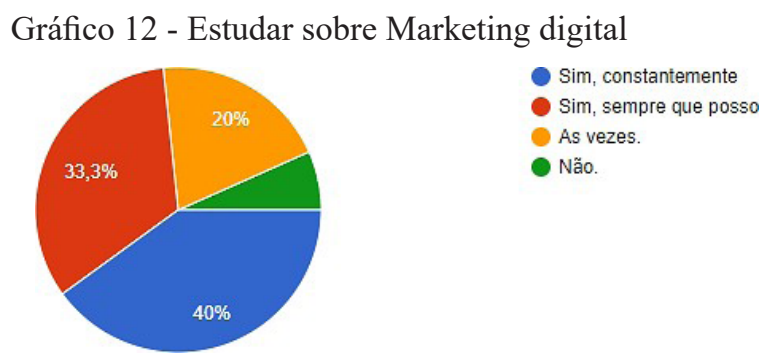

Fonte: Dados da pesquisa (2020).
Gráfico 13 - Meio que proporciona maiores vendas

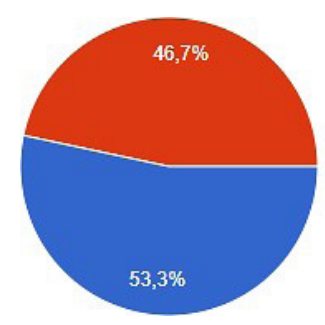

Fonte: Dados da pesquisa (2020). 


\section{CONCLUSÃO}

Com o objetivo de avaliar a utilização das mídias sociais das pequenas empresas, concluiu-se que o marketing digital, a partir das mídias digitais, tem sido uma ferramenta bastante poderosa de engajamento social, bem como comercial, principalmente, os meios digitais gratuitos como as redes sociais, o que proporciona inúmeras vantagens, especialmente, às micro e pequenas empresas alvo desta pesquisa.

Observou-se também que as pequenas empresas da cidade ainda estão acanhadas em relação aos investimentos em marketing digital, e principalmente em conhecimento de conteúdo e investimento financeiro. Portanto, levando em consideração a comprovada importância do marketing digital, os empreendedores têm a missão de desenvolver e alavancar o marketing digital das suas empresas para que logrem sucesso nos seus empreendimentos e não se contentarem-se apenas com meios padrões e convencionais de mídias digitais.

\section{REFERÊNCIAS}

FLICK, Uwe. Introdução à metodologia de pesquisa: um guia para iniciantes. Porto Alegre: Penso, 2013.

FRANCA, Geraldo. Marketing digital para pequenas e microempresas. Disponível em: http:// www.empreendedor.com.br/artigo/marketing-digital-para-pequenas-e-micro-empresas. Acesso em: 10 dez. 2011.

GANSKY, Lisa. Mesh: por que o futuro dos negócios é compartilhar. Rio de janeiro: Alta Books, 2011.

KOTLER, Philip. Marketing 4.0 [recurso eletrônico]. Rio de Janeiro: Sextante, 2017. Disponível em: http://professor.pucgoias.edu.br/SiteDocente/admin/arquivosUpload/17352/material/Marketi n-0-Do-tradicional-ao-digital\%20(1).pdf. Acesso em: 10 nov. 2020.

PRODANOV, Cleber Cristiano; FREITAS, Ernani César De. Metodologia do trabalho científico: métodos e técnicas da pesquisa e do trabalho acadêmico. 2. ed. Novo Hamburgo: Feevale, 2013.

SERVIÇO BRASILEIRO DE APOIO ÀS MICRO E PEQUENAS EMPRESAS (SEBRAE). Tecnologia da informação. Disponível em: http://www.informamidia. com.br/artigo-marketing-digital-para-pequenas-e-micro-empresas//. Acesso em: 10 nov. 2020.

TEIXEIRA, Rafael Farias. Especialista em Facebook diz como as pequenas empresas podem tirar proveito da rede social. Disponível em: http:/www.informamidia.com.br/artigo-marketing-digital-para-pequenas-e-micro empresas/. Acesso em: 12 set. 2020.

TORRES, Cláudio, Guia prático de marketing na internet para pequenas empresas: dicas para posicionar o seu negócio e conquistar novos clientes na Internet. 2010. Disponível em: http://www.in- 
formamidia.com.br/artigo-marketing-digital-para-pequenas-e- micro-empresas/. Acesso em: 10 nov. 2020.

TORRES, Cláudio. Guia Prático de Marketing na Internet para Pequenas Empresas. 2010. Disponível em: http://uab.ifsul.edu.br/tsiad/conteudo/modulo5/gne/biblioteca/claudio torres_mktdigitalpequenaempresa.pdf. Acesso em: 20 nov. 2020.

TWY, Carol. Os 4 pilares do marketing digital para pequenas empresas. 2012. Disponível em: http://www.informamidia.com.br/artigo-marketing-digital-para-pequenas-e-micro- empr esas/.Acesso em: 20 nov. 2020. 


\section{Índice Remissivo}

$\mathbf{A}$

ação planejada 46

acesso ao crédito 139,140

administração de empresas 29, 30, 31, 32, 33, 34, 35

administração escolar 216

ambiente organizacional 20,21, 174

ambientes educacionais 38

armazenagem dos produtos 99

ascensão social 29

aspecto logístico 112

atividade comercial 127

atividade econômica 38, 63, 198

atividades de consumo 139

atividades empreendedoras 38, 195

\section{B}

base gerencial sólida 46

C

campanhas publicitárias 118

capacidade de articulação 47

capacidades técnicas específicas 47

capacitação $35,38,58,59,66,154,191$

capitalismo no Brasil 46

capital parado 99,100

cenário mercadológico 172

cliente $88,90,93,99,107,108,112,113,114,115,116,118,119,120,121,133,134$

clientes e a empresa 112

comunicação $8,20,21,22,23,26,27,41,42,52,53,54,55,56,59,67,86,95,118,119,120,173,175,176,207,211$

comunicação de massa 21

comunicação empresarial 20,26

comunicação interpessoal 8,21

comunidade escolar 216, 218, 219, 221, 223, 224, 225

concorrência $60,85,92,133,189$

conectividade 118,119

conflitos étnicos 46

conflitos internos 20,21, 22, 23, 32

conquista do voto 29

conquista por cidadania 29

consumidor 71, 81, 115, 118, 119, 149

consumo descontrolado 139

controle de estoque 70,99 
controle de produtos 99

controle financeiro 139, 140, 143, 144

corrupção 46

credibilidade 32, 33, 148, 173

crescimento da população 139

crescimento das mídias 112

crescimento de mercado 58

criatividade $8,41,58,59,60,61,63,64,66,67,109,191$

crime organizado 46

crise sanitária do Covid19 194

crises inflacionárias 139

custo de armazenagem 99, 100, 104, 105, 106, 107, 108, 109

\section{D}

deficiência no gerenciamento 46

delivery 66,195

demanda 59, 70,71,74, 100, 114, 132, 136, 139, 194

desafios financeiros 139

desenvolver estratégias 47

desenvolvimento econômico brasileiro 59

desenvolvimento tecnológico 112,120

desigualdades sociais $33,46,63,149$

desregulamentação de políticas sociais 46

direito igualitário 29

direitos femininos 29

direitos humanos 46, 149

distribuidora de bebidas 99, 100, 109, 110

$\mathbf{E}$

educação financeira $8,139,140,141,142,143,144,145,160,161,162,165,166,168,170$

empoderamento feminino $29,30,31,32,33,34,35$

empreendedor 38, 39, 40, 41, 42, 43, 44, 58, 64, 65, 66, 88, 124, 194, 196, 198, 203

empreendedores brasileiros 38

empreendedores individuais 38,43

empreendedorismo no Brasil 31, 194

empregados com deficiências 147

empresa-alvo 128

empresas 29, 30, 32, 33, 38, 42, 43, 44, 59, 60, 61, 63, 65, 66, 67, 68, 70, 71, 85, 88, 89, 96, 97, 99, 107, 115, 118, 119, $120,121,122,123,124,125,128,147,149,150,151,152,153,154,155,156,157,158,164,172,173,194$, $195,200,203,206$

empresa varejista $85,87,96$

endividamento $139,140,141,142,143,144,145,167,168$

endividamento da população 139

ensino de qualidade 207

escolha do produto 112

esferas educacionais 207,216 
essência da administração 47

estagnação econômica 139

estilos de liderança 173

estoques competente 70

estratégia empresarial 59

estratégia organizacional $27,56,85$

estratégias de liderar 172

exclusão 147, 148, 217

expansão da mulher no mercado 29, 30, 32, 34, 35

expansão tecnológica 58

F

facilidade de crédito 139, 144, 164

feminino na sociedade contemporânea 29

ferramentas gerenciais 47

fidelização dos clientes 118

flexibilidade de horários 206

formação pessoal e profissional 35

forma remota 206, 209

funcionamento de estoque 100

funções básicas do administrador 47

G

geração de empregos 39

gerenciamento de matérias-primas 127

gerenciamento de recursos 139

gerenciamento do serviço logístico 112

gestão ao democrático/participativo 216

gestão da produção $8,127,130,133,136$

gestão de estoque $70,99,100,104,106,107,109$

gestão de estoques $70,71,72,83$

gestão democrática 216, 218, 219, 220, 221, 222, 223, 224, 225, 226

gestão do Terceiro Setor 47

gestão escolar 8, 216, 218, 219, 223, 225, 226

gestor $42,46,48,51,88,93,127,128,133,134,135,136,218,219,221,222,224,225,226$

gestores de produção 128

grupos competidores 61

H

Home Office 206, 207, 209, 210, 211, 213, 214

\section{I}

igualdade $29,34,149,150,151$

imagem institucional 47, 149

Indústria 21

inflação 58, 139 
inovação $8,31,43,58,59,60,61,62,63,64,65,66,67,68,90,112,151,214$

inovação organizacional 59, 60

instituições educacionais 216

instituições governamentais 38

$\mathbf{L}$

líder 46, 63, 172, 173, 175, 176

liderança $8,27,31,33,38,51,54,63,154,155,172,173,174,175,176,194,195,197,219,221,222,223$

líder de equipe 46

limitações 68, 91, 116, 144, 147, 153

luta das mulheres 29

lutas feministas 29

$\mathbf{M}$

marketing de relacionamento 119, 192

marketing digital 118, 119, 122, 123, 124, 125

marketing pessoal 8, 189, 190, 191, 192

mercado saturado 85

metas corporativas 20

mídias sociais 26, 42, 119, 120, 124

modalidade de Educação a Distância (EAD) 207

modelos de lideranças 172

modernização do mercado financeiro 140

modo de trabalho 206

movimento feminista 29

mudanças no regime de trabalho 195

$\mathbf{N}$

negócios 31, 38, 41, 42, 49, 50, 53, 63, 65, 67, 71, 119, 120, 124, 149, 194, 195, 196, 197, 198, 201, 202, 203

nicho de mercado 118, 194, 197

níveis de desemprego 139

nível de estoque adequado 70

$\mathbf{O}$

oferta de produtos 119

oferta do crédito 139

oportunidades no mercado de trabalho 147

organizações $8,21,27,34,35,38,42,46,47,49,51,53,56,61,71,90,93,99,103,121,147,148,151,152,156,191$, 214

organizações não governamentais (ONG's) 46

$\mathbf{P}$

pandemia $42,48,65,160,161,162,164,165,166,167,168,170,194,195,202,203,206,207,209,210,213$

panorama conceitual 30

papéis gerenciais 47

pequenas empresas $85,86,118,124$ 
pessoas de baixa renda 139

pessoas portadoras de deficiência 147,152

planejamento $8,42,47,49,51,53,54,56,65,85,86,87,88,89,90,93,94,96,97,115,116,127,128,130,133,135$, $136,139,140,143,144,151,154,155,156,157,160,162,166,190,213,218,219,222$

planejamento da gestão 8, 127, 136

planejamento de produção 128

planejamento estratégico $8,51,85,86,87,88,89,93,94,96,157$

poder de compra 139,167

prática de trabalho 207

prática empreendedora 38,39

preconceito $30,32,35,147,152,153$

preconceitos $29,31,32$

privatização de serviços essenciais 46

processo administrativo 47, 48, 54

processo de educação 216

processo de gestão 47, 49, 70

produtividade 26, 27, 59, 60, 97, 127, 152, 191, 206, 209, 213

Produto Interno Bruto (PIB) 139

produtos em estoque 99, 103

profissional especializado 118, 211

Projetare Social 47, 48, 49, 50, 51, 52, 53, 54

provedores de mercadorias 194

público-alvo 49, 118

Q

qualidade de serviços 47

qualidade de vida da sociedade 46

qualidade dos serviços 112, 207

quantidade de itens/produtos 99

questão social 147

$\mathbf{R}$

realidade contemporânea 59,61

recrutamento de pessoas 147, 148, 149, 150, 151, 154, 155, 156, 157

recrutamento de pessoas com deficiência 147, 149, 150, 151, 154, 155, 156, 157

recursos computacionais 207

recursos criativos 59

recursos duráveis ou não duráveis 70

Recursos Humanos (RH) 147

recursos tecnológicos 206, 211

redes sociais $112,118,121,122,123,124,162$

relações de gênero 29, 36

relações humanas 20,218

rendimentos pessoais 139

resistência feminina 29

revolução 4.058 
revolução tecnológica 58

$\mathbf{S}$

satisfação do cliente 112

satisfação do cliente após as vendas 112

satisfação no pós- venda 112

SEBRAE 11, 38, 44, 65, 68, 89, 97, 119, 124, 149, 158, 195, 203

segmentos da economia 58

Serviço Brasileiro de Apoio às Micro e Pequenas Empresas 38, 65, 119, 158, 195

serviço de cunho social 59

serviços bancários 139

setor alimentício 85,96

setor industrial 127

stakeholders 47

sucesso empresarial 172

sujeito criativo 38

supermercado $70,76,77,78,81,82$

suporte técnico-gerencial 58

sustentabilidade 47

T

tele trabalho 206, 209

tendências de mercado 60

terceiro setor 8, 46, 55

trabalho criativo 60

transparência $20,47,54$

V

vantagens competitivas 127

vendas online 195,200

viabilidade 59,144

visão estratégica 38

vivência diária 20 


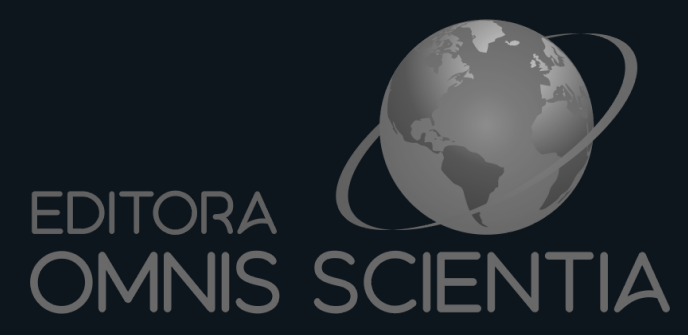

editoraomnisscientia@gmail.com $\square$ https://editoraomnisscientia.com.br/ (-) @editora_omnis_scientia @ https://www.facebook.com/omnis.scientia.9 f +55 (87) 9656-3565@ 


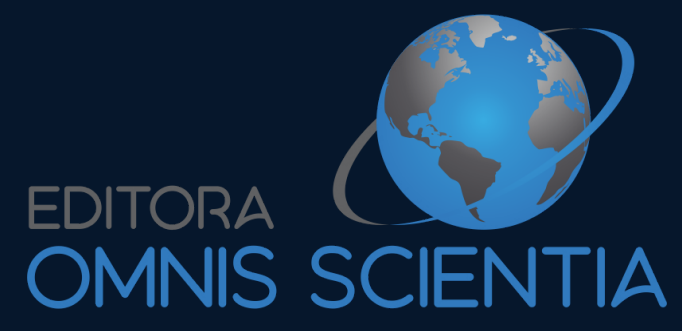

editoraomnisscientia@gmail.com $\square$ https://editoraomnisscientia.com.br/ 击

@editora_omnis_scientia (0) https://www.facebook.com/omnis.scientia.9 f +55 (87) 9656-3565 @ 